\title{
Management of Clear Cell Odontogenic Carcinoma: A Case Report
}

\author{
Pinelopi Theopisti Memtsa ${ }^{\mathrm{a}, \mathrm{c}}$, Aikaterini Papadopoulou ${ }^{\mathrm{a}}$, \\ Konstantinos Vachtsevanos ${ }^{\mathrm{b}}$, Ioannis Tzitzikas ${ }^{\mathrm{a}}$
}

\begin{abstract}
Clear cell odontogenic carcinoma (CCOC) is a low-grade odontogenic carcinoma showing cells with clear cytoplasm arranged in sheets and islands. Most of the CCOC typically present as swelling in anterior region of the mandible in elderly female patients. Radiographically, it exhibits ill-defined unilocular or multilocular radiolucency with tendency to cause bone destruction and tooth resorption. The present case report discusses the clinical, histological and immunohistochemical characteristics of a CCOC which was treated with surgery and adjuvant radiation therapy.
\end{abstract}

Keywords: Radiation therapy; Odontogenic carcinoma; Clear cell carcinoma

\section{Introduction}

Clear cell odontogenic carcinoma (CCOC) is a rare intraosseous carcinoma of the mandible which was first described as a clear cell odontogenic tumor in 1985 by Hansen. CCOC was initially known as clear cell odontogenic tumor or clear cell ameloblastoma. In 1992, CCOC was classified as odontogenic tumor by the WHO however, due to its aggressive tendency with local recurrence, regional lymph node metastasis, and distant metastasis, CCOC was considered to be a malignant tumor of odontogenic origin in the WHO classification of 2005 [1].

CCOC occurs predominantly in the fifth to seventh decades in women in the mandible. Painless swelling is the most common symptom and pain, teeth loosening and paresthesia follow.

CCOC is also difficult to diagnose histopathologically.

Manuscript submitted May 7, 2018, accepted May 23, 2018

aDepartment of Radiation Oncology, AHEPA University Hospital, Aristotle University of Thessaloniki, Greece

bepartment of Oral Maxillofacial Surgery, Aristotle University of Thessaloniki, Greece

${ }^{\mathrm{c} C}$ Corresponding Author: Pinelopi Theopisti Memtsa, Department of Radiation Oncology, AHEPA University Hospital, Aristotle University of Thessaloniki, Greece. Email: pennymemtsa@hotmail.com

doi: https://doi.org/10.14740/jmc3080w
The differential diagnosis of jaw tumors with prominent cytoplasmic clearing includes intraosseous salivary gland tumors (epithelial-myoepithelial carcinoma) and metastatic tumors like clear cell renal cell carcinoma. Other odontogenic tumors may also show clearing of their constituent cells. Such tumors include calcifying epithelial odontogenic tumor and clear cell ameloblastoma. While the former is identified by the presence of psammomatous calcifications and amyloid deposits, the latter may be difficult to distinguish from $\mathrm{CCOC}$ [2].

In $\mathrm{CCOC}$, surgical resection with a wide margin is the treatment of choice. Thus, proper jaw reconstruction is important and should be performed simultaneously with resection. Fibular free flap reconstruction is necessary when the resected jaw defect is large in the mandible. It provides several advantages over other donor sites, including adequate bone length, ease of graft dissection and contouring, a two-team approach, long pedicles with proper vessels and minimal donor site morbidity [3].

The present case report discusses the clinical, histological and immunohistochemical characteristics of a $\mathrm{CCOC}$ which was treated with surgery and adjuvant radiation therapy, and we review the literature for this rare tumor.

\section{Case Report}

A 64-year-old woman was referred to our hospital with a history of hyalinizing clear cell carcinoma of the upper right maxilla since 1999. She suffered from painless swelling and loose teeth in the right maxillary region. For that reason, she underwent a surgical excision. According to the histological report, it was a stage I hyalinizing clear cell maxillary carcinoma, without regional extension. The patient received adjuvant concurrent chemoradiotherapy with cisplatin/5-fluorouracil for a total of six cycles along with external beam radiation therapy with a dose of $50 \mathrm{~Gy}$. She has been asymptomatic since then and free of recurrence 4 years postoperatively on regular follow-up with enhanced computed tomography (CT). There was a bone deficiency in the right maxilla and right maxillary sinus due to surgery excision. In the anterior limits of the bone deficiency, there was a soft tissue mass which probably corresponded to the development of the fibrotic tissue in the region. In the context of her follow-up, the patient underwent a CT of head and neck in 2013, where was demonstrated a progressive increase in the size of the mass. 


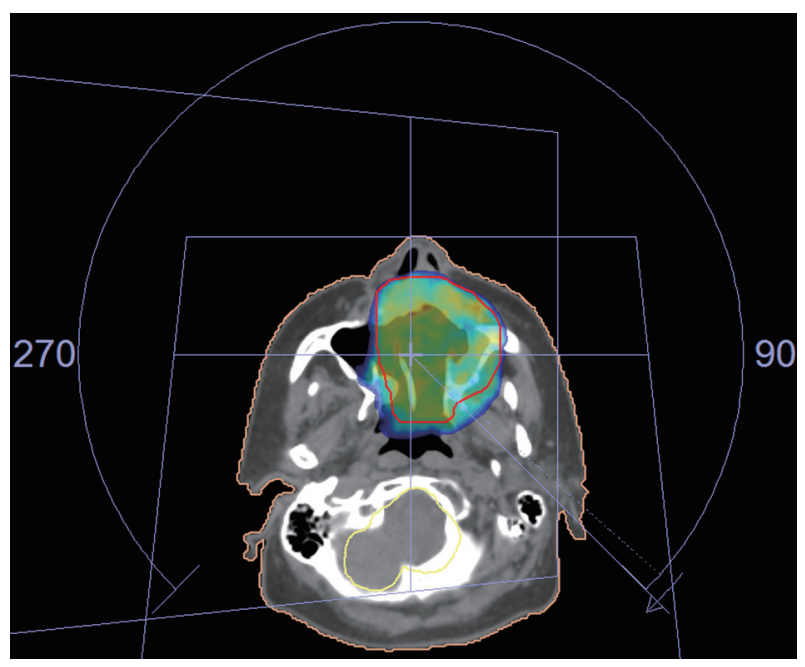

Figure 1. The radiotherapy fields for the treatment of the tumor.

Although the incisional biopsy revealed local recurrence, the removal part of right maxilla and maxillary sinus was negative for malignancy. Further follow-up evaluation including CT of head and neck, chest and abdomen demonstrated no evidence of local recurrence or distant metastasis. Three years later, during the follow-up period, a CT showed the presence of a mass measuring $2.6 \times 2 \mathrm{~cm}$, centered within the left osseous of the maxilla and left maxillary sinus involving the inferior nasal septum with extension into nasal canal. Under general anesthesia, the patient underwent a right partial maxillectomy with resection of the inferior nasal concha extended to pterygoid process via WeberFerguson approach. Surgery with negative margins was not possible.

Macroscopic examination on the surface of the mucosa revealed an alteration with focal ulceration measuring $3.4 \mathrm{~cm}$ with mildly abnormal limits and whitish appearance. Microscopic examination revealed a tumor consisted of medium/ large-sized cells with clear cytoplasm while few cells exhibited eosinophilic cytoplasm especially at the periphery of the tumor, which included eccentrically located sub-rounded small dark-staining nuclei with rare mitotic activity. These cells were with a vascularized and hyalinized stroma, arranged in sheets and islands. Bone congestion and discreet mononuclear inflammatory infiltrate were also observed. Perineural infiltration was noted without necrosis.

On immunohistochemical staining, the following immunophenotypes were found: $\mathrm{Ck} 8 / 18++, 34 \mathrm{BE} 12+$, p63++/-, Ck 19++, Ck 7+/-, Ck5/6 +/-, SMA - and CD 10-. The Ki-67 cell proliferation index was about $5-6 \%$; thus, the lesion indicated a low-grade tumor. Periodic acid Schiff (PAS) stain and PAS-diastase stains were positive in some neoplastic cells for cytoplasmic glycogen, and more rarely in other cells for cytoplasmic mucus. The tumor cells had connection with the overlying squamous epithelium, which was focally ulcerated. These findings were consistent wotj the diagnosis of hyalinizing clear cell carcinoma of maxilla.

One month after surgery, she attended to our Radiotherapy Department for adjuvant radiation therapy, due to close surgi- cal margin. The radiotherapy was performed in a linear accelerator with 6-MV energy, with VMAT delivery technique. She received a total dose of 66 Gy in 33 fractions with a daily dose of $2 \mathrm{~Gy}$ in the tumor bed (Fig. 1).

Acute radiation mucositis and dermatitis were observed, both of which were classified as grade 2 . The patient was followed up 1 month later with clinical examination and her mucositis and dermatitis were resolved.

\section{Discussion}

The term clear cell odontogenic tumor was coined by Hansen et al. CCOC is a rare neoplasm with only few cases reported in the literature. Clear cell lesions in the head and neck region have a wide range of differential diagnosis that includes odontogenic tumors such as ameloblastoma, calcifying epithelial odontogenic tumor, odontogenic carcinoma and salivary gland tumors like mucoepidermoid carcinoma or hyalinizing clear cell carcinoma. In 2005, WHO classified CCOC as malignant tumors characterized by sheets and islands of vacuolated and clear cells. Its local aggressive growth, frequent recurrence and occasional metastasis are reported in several cases [4].

CCOC primarily affects females. The majority of cases have been diagnosed in patients older than 40 years. The peak incidence is the fifth to seventh decades. According to the literature, 73 cases of CCOC have been reported with the mandible being more commonly affected than the maxilla. The clinical presentation of CCOC has been reported to present as a painless swelling. Pain and regional tooth mobility are only occasionally present.

Histologically, CCOC was defined as "a benign but locally invasive neoplasm originating from odontogenic epithelium and characterized by sheets, cords and islands of monomorphic, round to oval vacuolated and clear cells".

Clear cells are hallmark but not the pathognomonic feature of CCOC. They are noted in odontogenic, salivary, melanotic, renal, thyroid and prostate tumors. Thus, the differential diagnosis of CCOC evokes a broad range of tumors with diverse histogenesis and biological behavior. The differentiation of these tumors is confounding because of the considerable overlap in their histological features, thus posing a diagnostic challenge. The differential diagnoses for CCOC include clear cell variant of calcifying epithelial odontogenic tumor (CCCEOT), ameloblastic carcinoma, intraosseous variant of clear cell mucoepidermoid carcinoma (CCMEC), hyalinizing clear cell carcinoma (CCC), intraosseous variant of melanoma, and metastatic tumors from kidney, thyroid and prostate [5].

Tumor cells of CCOC exhibit prominent diastase-digested PAS-positive granules. PAS-positive granules show intracytoplasmic glycogen deposition. They are immunoreactive for cytokeratin 8 and cytokeratin 19, Ki-67, carcinoembryonic antigen (EMA), epithelial membrane antigen (CEA) and nonreactive for S-100 protein, glial fibrillary acidic protein, involucrin, vimentin and smooth muscle actin [6].

Radiographic images of CCOC generally demonstrate ra- 
diolucency but occasionally they are mixed. The differential diagnosis is broad, so a careful approach is necessary both clinically and immunohistochemically [7].

The clinical and biological behaviors of CCOC are complicated by the different prognoses and mortality rates reported in the scientific literature. The aggressiveness of these neoplasms are documented as extensive invasion of adjacent tissues, regional metastasis to the lymph nodes, less frequent distant metastasis to the lungs and a recurrence rate of $55 \%$. The management of CCOC is a challenge as the most appropriate treatment for a tumor is determined by a definitive diagnosis and adequate understanding of the biologic behavior of the tumor. Because of the insufficiency in the number of cases and the varied prognosis reported, various therapeutic approaches have been applied by the surgeons over the years including curettage, enucleation, en bloc resection and subtotal mandibulectomy/maxillectomy. Recurrence (80\%) within 2 years and/or metastasis developed in cases, which underwent curettage or enucleation, and hence, wide local resection with partial mandibulectomy/maxillectomy with clear margins was the treatment of choice. Fatal clinical outcome has been reported in cases with distant metastasis. Treatment protocol included lymph node resection and radical surgery if the nodes were positive, and adjuvant chemotherapy and/or radiotherapy for those with tumor positive margins and/or regional/neural/vascular invasion [8]. Adjuvant radiotherapy at carcinoma doses of 66 Gy at 2 Gy per fraction delivered once a day or a total dose of 74 Gy at 1.2 Gy per fraction delivered twice daily is advocated by some authors. Chemotherapy is usually reserved for palliative care as there is lack of evidence for its efficacy in definitive treatment [9]. CCOC is a rare odontogenic tumor and very few cases have been reported in the literature. Differentiation from other clear cell lesions is very important. Further studies on clinical, histological and immunohistochemical properties of this tumor need to be considered. Also, due to its aggressive nature, long-term follow-up is mandatory.

\section{References}

1. Maiorano E, Altini M, Viale G, Piattelli A, Favia G. Clear cell odontogenic carcinoma. Report of two cases and review of the literature. Am J Clin Pathol. 2001;116(1):107-114.

2. Zhang J, Liu L, Pan J, Tian X, Tan J, Zhou J, Duan Y. Clear cell odontogenic carcinoma: report of 6 cases and review of the literature. Med Oncol. 2011;(Suppl 1):S626-633.

3. Avninder S, Rakheja D, Bhatnagar A. Clear cell odontogenic carcinoma: a diagnostic and therapeutic dilemma. World J Surg Oncol. 2006;4:91.

4. Eversole LR, Belton CM, Hansen LS. Clear cell odontogenic tumor: histochemical and ultrastructural features. J Oral Pathol. 1985;14(8):603-614.

5. Elbeshir EI, Harris M, Barrett AW. Clear cell odontogenic carcinoma of the maxilla: Clinical, histological and immunohistochemical features of a case. Oral Oncology Extra. 2004;40:91-94.

6. Li TJ, Yu SF, Gao Y, Wang EB. Clear cell odontogenic carcinoma: a clinicopathologic and immunocytochemical study of 5 cases. Arch Pathol Lab Med. 2001;125(12):1566-1571.

7. Krishnamoorthy R, Ravi Kumar AS, Batstone M. FDG$\mathrm{PET} / \mathrm{CT}$ in staging of clear cell odontogenic carcinoma. Int J Oral Maxillofac Surg. 2014;43(11):1326-1329.

8. Bilodeau EA, Hoschar AP, Barnes EL, Hunt JL, Seethala RR. Clear cell carcinoma and clear cell odontogenic carcinoma: a comparative clinicopathologic and immunohistochemical study. Head Neck Pathol. 2011;5(2):101-107.

9. Infante-Cossio P, Torres-Carranza E, Gonzalez-Perez LM, Gonzalez-Cardero E, Sanchez-Gallego F. Atypical presentation of clear cell odontogenic carcinoma. J Craniofac Surg. 2012;23(5):e466-468. 